\title{
Cold three-level atom micromaser in the far-detuning regime
}

\author{
John Martin and Thierry Bastin* \\ Institut de Physique Nucléaire, Atomique et de Spectroscopie, Université de Liège au Sart Tilman, Bâtiment B15, B-4000 Liège, Belgium
}

(Received 13 December 2006; published 24 May 2007)

\begin{abstract}
We analyze the quantum theory of the two-mode micromaser pumped by cold three-level atoms with a $\Lambda$-configuration under the two-photon resonance condition. We focus more specifically on the large detuning limit where the detuning between the two cavity mode frequencies and the two atomic transition frequencies is large in comparison with the atom-cavity coupling constants. We show that this regime can mimic a virtual two-level atom micromaser without spontaneous emission where the cavity acts for the atoms as a potential barrier or a potential well (depending on the sign of the detuning) and a zero potential but no more both as a potential barrier and a potential well as it is the case for the usual cold two-level atom micromaser [M. O. Scully, G. M. Meyer, and H. Walther, Phys. Rev. Lett. 76, 4144 (1996)]. This introduces interesting options for engineering the interaction between the cold atoms and the cavity. The elimination of the spontaneous emission solves a major issue of the conventional cold two-level atom micromaser as the transit time of cold atoms inside and in the vicinity of the cavity is usually much larger than the atomic level lifetimes. It is also shown that the cold atom regime is very sensitive to the sign of the detuning (in contrast to the hot atom regime) and that, according to this sign, the cavity may speed up or slow down the incident three-level atoms after their interaction with the field.
\end{abstract}

DOI: $10.1103 /$ PhysRevA.75.053820

PACS number(s): 42.50.Pq, 42.50.Vk, 32.80.Lg

\section{INTRODUCTION}

Today, laser cooling of atoms has become a very broad topic in atomic physics and quantum optics. Cold and ultracold atoms introduce new regimes often not considered in the past. Particularly, the study of the micromaser exhibits important new effects when cold atoms are sent through the maser cavity rather than hot ones [1]. In this case, Englert et al. [2] have shown that the cavity acts simultaneously for the incident atoms as a potential barrier and a potential well, even if the cavity contains no photon. This effect arises from the sudden change of the atom-field interaction when the atoms enter the cavity. It requires the quantization of the center-of-mass motion to be explained. For this reason, the system has been called mazer by Scully and co-workers [1] to insist on the fact that the microwave amplification is due to the $z$ motion axis induced emission of radiation.

In the last decade, a lot of studies were dedicated to this subject (see, e.g., references in [3]). Initially, two-level atoms interacting with the cavity were considered [4-6]. Later on, models using three-level atoms have been developed [7-15]. Zhang et al. [7-10] focused on three-level atoms in $\Lambda$ and $\Xi$ configurations interacting with monomodal cavities. Liang et al. [11] investigated the transmission probability of $V$-type three-level atoms. Arun et al. $[12,13]$ considered the case of three-level atoms in $\Lambda$ and $\Xi$ configurations interacting resonantly with a bimodal cavity (each transition frequency coincides with a mode frequency). Abdel-Aty and Obada $[14,15]$ studied transient phenomena during the passage of the atoms through the cavity.

In this paper, we consider $\Lambda$-type three-level atoms interacting with a bimodal cavity in the general two-photon resonance condition (see Fig. 1). We focus more specifically on

\footnotetext{
*Electronic address: t.bastin@ulg.ac.be
}

the large detuning limit where the detuning $\Delta$ between the two cavity mode frequencies and the two atomic transition frequencies is large in comparison with the atom-cavity coupling constants. In the hot atom regime where the quantization of the atomic motion is not required, it is well known that the large-detuning limit simplifies the atom-cavity interaction to the interaction of a virtual two-level atom with a monomodal cavity $[16,17]$. In the cold atom regime, we show here that this is more subtle. Still the atom behaves like a virtual two-level one. However, the two-level atom mazer properties are not entirely recovered. The large-detuning limit of the three-level atom mazer mimics a two-level atom mazer where the cavity acts for the atoms initially in one of their two lower states as a potential barrier or a potential well (depending on the sign of the detuning) and a zero potential but no more both as a potential barrier and a potential well as it is the case for the usual two-level atom mazer $[1,2,4]$. This changes drastically the properties of the system and introduces new interesting options for engineering the interaction between the atoms and the cavity as the sole detuning parameter determines now what kind of potential the atoms interact with and also directly the potential height or depth. In the conventional mazer, no such degree of freedom exists since both mechanical potentials are always present. Also, similarly to three-level atoms interacting with two traveling waves [18], the large-detuning limit allows a system where the spontaneous emission is virtually eliminated when the two lower levels do not define an allowed dipolar transition. This is particularly important in the cold atom regime where the transit times inside and in the vicinity of the cavity can be much larger than the atomic level lifetimes.

The paper is organized as follows. In Sec. II, the Hamiltonian modeling a $\Lambda$-type three-level atom interacting with a bimodal cavity in the general two-photon resonance condition is presented and the wave functions of the system are described. General expressions for the transition probabilities between the different atomic levels are given. We prove there 
that, similarly to two-level atoms interacting nonresonantly with a monomodal cavity [3], the cavity may slow down or speed up the atoms according to the sign of the detuning $\Delta$. We then focus on the large detuning limit to show that the interaction of the three-level atoms with the bimodal cavity leads to a one-dimensional scattering process upon a potential barrier or a potential well. Simplified expressions for the transition probabilities are given. In Sec. III, we discuss more specifically the properties of these transition probabilities and make the connection to the hot atom regime where the quantization of the atomic motion is not needed. Experimental considerations are given in Sec. IV. A brief summary of our results is finally given in Sec. V.

\section{MODEL}

\section{A. The Hamiltonian}

\section{General expression}

We consider a $\Lambda$-type three-level atom (with levels $\left|b_{1}\right\rangle$, $\left|b_{2}\right\rangle$, and $|a\rangle$ of energy $\hbar \Omega_{b_{1}}, \hbar \Omega_{b_{2}}$, and $\hbar \Omega_{a}$, respectively) moving along the $z$ direction on the way to a bimodal cavity defined to be located in the range $0<z<L$ (see Fig. 1). We denote by $\omega_{1}$ and $\omega_{2}$ the two mode frequencies. We assume no coupling between the two lower levels $\left|b_{1}\right\rangle$ and $\left|b_{2}\right\rangle$ and the two-photon resonance condition $\Delta \equiv \omega_{1}-\left(\Omega_{a}-\Omega_{b_{1}}\right)=\omega_{2}$ $-\left(\Omega_{a}-\Omega_{b_{2}}\right)$ is fulfilled. The atomic center-of-mass motion is described quantum mechanically and the usual rotating-wave approximation is made. The Hamiltonian of this system is given by

$$
H=H_{A}+H_{F}+H_{A F},
$$

where $H_{A}\left(H_{F}\right)$ is the Hamiltonian of the free atom (field) and $H_{A F}$ is the interaction Hamiltonian describing the atomfield interaction, with

$$
\begin{gathered}
H_{A}=\frac{p^{2}}{2 m}+\hbar \Omega_{a}|a\rangle\left\langle a\left|+\sum_{\alpha=1}^{2} \hbar \Omega_{b_{\alpha}}\right| b_{\alpha}\right\rangle\left\langle b_{\alpha}\right|, \\
H_{F}=\sum_{\alpha=1}^{2} \hbar \omega_{\alpha} a_{\alpha}^{\dagger} a_{\alpha}, \\
H_{A F}=\sum_{\alpha=1}^{2} \hbar g_{\alpha} u_{\alpha}(z)\left(a_{\alpha}|a\rangle\left\langle b_{\alpha}|+| b_{\alpha}\right\rangle\langle a| a_{\alpha}^{\dagger}\right) .
\end{gathered}
$$

In Eq. (2), $p$ is the atomic center-of-mass momentum along the $z$ axis, $m$ is the atomic mass, $|j\rangle\langle j|\left(j=a, b_{1}, b_{2}\right)$ are the projection operators onto the states $|j\rangle,|i\rangle\langle j|\left(i, j=a, b_{1}, b_{2}\right.$; $i \neq j$ ) are the transition operators from level $j$ to level $i, a_{\alpha}$ and $a_{\alpha}^{\dagger}(\alpha=1,2)$ are, respectively, the annihilation and creation operators of a photon in mode $\alpha, g_{\alpha}$ are the atom-field coupling strengths at the two mode frequencies $\omega_{\alpha}$, and $u_{\alpha}(z)$ are the cavity field mode functions, which are assumed here to be identical, i.e., $u_{1}(z)=u_{2}(z) \equiv u(z)$. In the following, we denote the cavity field eigenstates of the two modes by $\left|n_{1}, n_{2}\right\rangle$ and the global state of the atom-field system at any time $t$ by $|\psi(t)\rangle$.

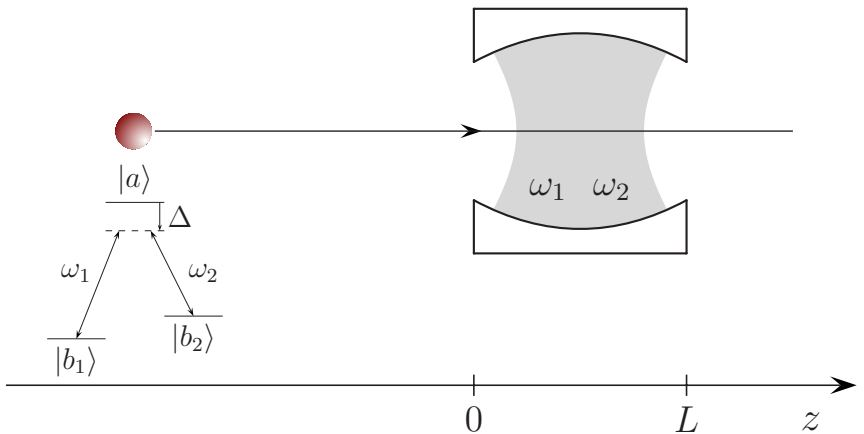

FIG. 1. (Color online) System investigated: A $\Lambda$-type three-level atom (with levels $\left|b_{1}\right\rangle,\left|b_{2}\right\rangle$, and $|a\rangle$ of energy $\hbar \Omega_{b_{1}}, \hbar \Omega_{b_{2}}$, and $\hbar \Omega_{a}$, respectively) moving along the $z$ direction on the way to a bimodal cavity of length $L$ (with mode frequencies $\omega_{1}$ and $\omega_{2}$ ) in the general two-photon resonance condition $\Delta \equiv \omega_{1}-\left(\Omega_{a}-\Omega_{b_{1}}\right)$ $=\omega_{2}-\left(\Omega_{a}-\Omega_{b_{2}}\right)$.

\section{Effective two-level Hamiltonian}

If the atomic upper level $|a\rangle$ is initially not populated and if the detuning $\Delta$ is large in comparison with the Rabi frequencies $\Omega_{n_{\alpha}} \equiv 2 g_{\alpha} \sqrt{n_{\alpha}+1}$ associated to the occupation numbers $n_{\alpha}$ of the field state, then the three-level system reduces to a virtual two-level one (comprising the $\left|b_{1}\right\rangle$ and $\left|b_{2}\right\rangle$ states) described in the interaction picture by the effective Hamiltonian [19]

$$
\begin{aligned}
H_{\text {eff }}= & \frac{p^{2}}{2 m}+\sum_{\alpha=1}^{2} \hbar \frac{g_{\alpha}^{2} u_{\alpha}^{2}(z)}{\Delta} a_{\alpha}^{\dagger} a_{\alpha}\left|b_{\alpha}\right\rangle\left\langle b_{\alpha}\right| \\
& +\hbar \frac{g_{1} u_{1}(z) g_{2} u_{2}(z)}{\Delta}\left(a_{1}^{\dagger} a_{2}\left|b_{1}\right\rangle\left\langle b_{2}\left|+a_{2}^{\dagger} a_{1}\right| b_{2}\right\rangle\left\langle b_{1}\right|\right) .
\end{aligned}
$$

\section{B. Wave functions}

\section{Arbitrary detuning}

Outside the cavity, the mode function $u(z)$ vanishes and the Schrödinger equation $i \hbar d / d t|\psi(t)\rangle=H|\psi(t)\rangle$ reads in the $z$ representation and in the noncoupled state basis

$$
\begin{gathered}
i \hbar \frac{\partial}{\partial t} \psi_{n_{1}, n_{2}}^{a}(z, t)=\left(-\frac{\hbar^{2}}{2 m} \frac{\partial^{2}}{\partial z^{2}}-\hbar \Delta\right) \psi_{n_{1}, n_{2}}^{a}(z, t), \\
i \hbar \frac{\partial}{\partial t} \psi_{n_{1}+1, n_{2}}^{b_{1}}(z, t)=-\frac{\hbar^{2}}{2 m} \frac{\partial^{2}}{\partial z^{2}} \psi_{n_{1}+1, n_{2}}^{b_{1}}(z, t), \\
i \hbar \frac{\partial}{\partial t} \psi_{n_{1}, n_{2}+1}^{b_{2}}(z, t)=-\frac{\hbar^{2}}{2 m} \frac{\partial^{2}}{\partial z^{2}} \psi_{n_{1}, n_{2}+1}^{b_{2}}(z, t),
\end{gathered}
$$

with

$$
\begin{gathered}
\psi_{n_{1}, n_{2}}^{a}(z, t)=e^{i E_{b} t / \hbar}\left\langle z, a, n_{1}, n_{2} \mid \psi(t)\right\rangle, \\
\psi_{n_{1}+1, n_{2}}^{b_{1}}(z, t)=e^{i E_{b} t / \hbar}\left\langle z, b_{1}, n_{1}+1, n_{2} \mid \psi(t)\right\rangle, \\
\psi_{n_{1}, n_{2}+1}^{b_{2}}(z, t)=e^{i E_{b} t / \hbar}\left\langle z, b_{2}, n_{1}, n_{2}+1 \mid \psi(t)\right\rangle .
\end{gathered}
$$

where 


$$
\begin{aligned}
E_{b} & =\hbar \Omega_{b_{1}}+\left(n_{1}+1\right) \hbar \omega_{1}+n_{2} \hbar \omega_{2} \\
& =\hbar \Omega_{b_{2}}+n_{1} \hbar \omega_{1}+\left(n_{2}+1\right) \hbar \omega_{2}
\end{aligned}
$$

is the energy of the atom-field states $\left|b_{1}, n_{1}+1, n_{2}\right\rangle$ and $\left|b_{2}, n_{1}, n_{2}+1\right\rangle$. In Eq. (5), we merely shift the energy scale origin at the $\left|b_{1}, n_{1}+1, n_{2}\right\rangle$ and $\left|b_{2}, n_{1}, n_{2}+1\right\rangle$ level.

The solutions to Eqs. (4) are obviously given by linear combinations of plane wave functions. If we assume initially a monokinetic atom (with momentum $\hbar k$ ) coming upon the cavity from the left-hand side (negative $z$ values) in the state $\left|b_{1}\right\rangle$ and the cavity field in the number state $\left|n_{1}+1, n_{2}\right\rangle$, the atom-field system is described outside the cavity by the wave function components (which correspond to the eigenstate $\left|\phi_{k}\right\rangle$ of energy $\left.E_{k}=\hbar^{2} k^{2} / 2 m\right)$

$$
\begin{gathered}
\psi_{n_{1}, n_{2}}^{a}(z, t)=e^{-i\left(\hbar k^{2} / 2 m\right) t} \varphi_{n_{1}, n_{2}}^{a}(z), \\
\psi_{n_{1}+1, n_{2}}^{b_{1}}(z, t)=e^{-i\left(\hbar k^{2} / 2 m\right) t} \varphi_{n_{1}+1, n_{2}}^{b_{1}}(z), \\
\psi_{n_{1}, n_{2}+1}^{b_{2}}(z, t)=e^{-i\left(\hbar k^{2} / 2 m\right) t} \varphi_{n_{1}, n_{2}+1}^{b_{2}}(z),
\end{gathered}
$$

with

$$
\begin{gathered}
\varphi_{n_{1}, n_{2}}^{a}(z)= \begin{cases}\rho_{n_{1}, n_{2}}^{a} e^{-i k_{a} z}, & z<0 \\
\tau_{n_{1}, n_{2}}^{a} e^{i k_{a} z}, & z>L,\end{cases} \\
\varphi_{n_{1}+1, n_{2}}^{b_{1}}(z)= \begin{cases}e^{i k z}+\rho_{n_{1}+1, n_{2}}^{b_{1}} e^{-i k z}, & z<0 \\
\tau_{n_{1}+1, n_{2}}^{b_{1}} e^{i k z}, & z>L,\end{cases} \\
\varphi_{n_{1}, n_{2}+1}^{b_{2}}(z)= \begin{cases}\rho_{n_{1}, n_{2}+1}^{b_{2}} e^{-i k z}, & z<0 \\
\tau_{n_{1}, n_{2}+1}^{b_{2}} e^{i k z}, & z>L,\end{cases}
\end{gathered}
$$

and

$$
k_{a}^{2}=k^{2}+\frac{2 m}{\hbar} \Delta \equiv k^{2}+\kappa^{2} \widetilde{\Delta}
$$

where

$$
\kappa^{2}=\frac{2 m}{\hbar} \sqrt{g_{1}^{2}+g_{2}^{2}}, \quad \tilde{\Delta}=\frac{\Delta}{\sqrt{g_{1}^{2}+g_{2}^{2}}} .
$$

The solutions (6) must be interpreted as follows: the atom initially in the state $\left|b_{1}\right\rangle$ coming upon the cavity will be found reflected in the state $|i\rangle\left(i=a, b_{1}, b_{2}\right)$ with the amplitude $\rho_{n_{1}, n_{2}}^{a}, \rho_{n_{1}+1, n_{2}}^{b_{1}}$, and $\rho_{n_{1}, n_{2}+1}^{b_{2}}$, respectively, or transmitted with the amplitude $\tau_{n_{1}, n_{2}}^{a}, \tau_{n_{1}+1, n_{2}}^{b_{1}}$ or $\tau_{n_{1}, n_{2}+1}^{b_{2}}$. However, in contrast to the resonant case $(\Delta=0$, see [12]), the atom reflected or transmitted in the excited state $|a\rangle$ will be found to propagate with a momentum $\hbar k_{a}$ different from its initial value $\hbar k$. The atomic transition $\left|b_{1}\right\rangle \rightarrow|a\rangle$ induced by the cavity is responsible for a change of the atomic kinetic energy. According to the sign of the detuning [see Eq. (10)], the cavity will either speed up the atom (for $\Delta>0$ ) or slow it down (for $\Delta<0$ ). This results merely from energy conservation. When, after leaving the cavity region, the atom is passed from the lower state $\left|b_{1}\right\rangle$ to the upper state $|a\rangle$, the photon number in mode 1 has decreased by one unit in the cavity and the internal energy of the atom-field system has varied by the quantity $\hbar\left(\Omega_{a}-\Omega_{b_{1}}\right)-\hbar \omega_{1}=-\hbar \Delta$. This variation needs to be exactly counterbalanced by the external energy of the system, i.e., the atomic kinetic energy. After the process, this energy has varied accordingly by the amount $\hbar \Delta$ (this is exactly the same effect that is observed for the nonresonant mazer with two-level atoms [3]). If the initial atomic kinetic energy $\hbar^{2} k^{2} / 2 m$ is lower than $\hbar|\Delta|$, and $\Delta<0$, the transition $\left|b_{1}, n_{1}+1, n_{2}\right\rangle \rightarrow\left|a, n_{1}, n_{2}\right\rangle$ cannot take place and this process is completely blocked.

Due to this change in the kinetic energy when the atom undergoes a transition from state $\left|b_{1}\right\rangle$ to state $|a\rangle$, the reflection and transmission probabilities of the atom in the excited state $|a\rangle$ are, respectively, given by

$$
\begin{aligned}
& R_{n_{1}, n_{2}}^{a}=\frac{k_{a}}{k}\left|\rho_{n_{1}, n_{2}}^{a}\right|^{2}, \\
& T_{n_{1}, n_{2}}^{a}=\frac{k_{a}}{k}\left|\tau_{n_{1}, n_{2}}^{a}\right|^{2},
\end{aligned}
$$

provided $\hbar^{2} k^{2} / 2 m>\hbar|\Delta|$ if $\Delta<0$. Otherwise, these probabilities vanish. The total atomic transition probability $\mathcal{P}_{b_{1} \rightarrow a}$ from state $\left|b_{1}\right\rangle$ to state $|a\rangle$ follows immediately:

$$
\mathcal{P}_{b_{1} \rightarrow a}=R_{n_{1}, n_{2}}^{a}+T_{n_{1}, n_{2}}^{a}
$$

that is

$$
\mathcal{P}_{b_{1} \rightarrow a}=\left\{\begin{array}{l}
\frac{k_{a}}{k}\left(\left|\rho_{n_{1}, n_{2}}^{a}\right|^{2}+\left|\tau_{n_{1}, n_{2}}^{a}\right|^{2}\right) \quad \text { if }\left(\frac{k}{\kappa}\right)^{2}+\tilde{\Delta}>0 \\
0 \quad \text { otherwise. }
\end{array}\right.
$$

When the atom remains in the lower state $\left|b_{1}\right\rangle$ or undergoes a transition to state $\left|b_{2}\right\rangle$ after having interacted with the cavity, there is no change in the atomic kinetic energy and the corresponding reflection and transmission probabilities are directly given by

$$
\begin{aligned}
& R_{n_{1}+1, n_{2}}^{b_{1}}=\left|\rho_{n_{1}+1, n_{2}}^{b_{1}}\right|^{2}, \\
& T_{n_{1}+1, n_{2}}^{b_{1}}=\left|\tau_{n_{1}+1, n_{2}}\right|^{2},
\end{aligned}
$$

and

$$
\begin{aligned}
& R_{n_{1}, n_{2}+1}^{b_{2}}=\left|\rho_{n_{1}, n_{2}+1}^{b_{2}}\right|^{2}, \\
& T_{n_{1}, n_{2}+1}^{b_{2}}=\left|\tau_{n_{1}, n_{2}+1}^{b_{2}}\right|^{2},
\end{aligned}
$$

respectively. The total probabilities to find the atom in the state $\left|b_{1}\right\rangle$ or $\left|b_{2}\right\rangle$ after its interaction with the cavity are accordingly given by

$$
\mathcal{P}_{b_{1} \rightarrow b_{1}}=\left|\rho_{n_{1}+1, n_{2}}^{b_{1}}\right|^{2}+\left|\tau_{n_{1}+1, n_{2}}^{b_{1}}\right|^{2}
$$

and

$$
\mathcal{P}_{b_{1} \rightarrow b_{2}}=\left|\rho_{n_{1}, n_{2}+1}^{b_{2}}\right|^{2}+\left|\tau_{n_{1}, n_{2}+1}^{b_{2}}\right|^{2},
$$

respectively. 
To determine any of the probabilities (12)-(18) the Schrödinger equation must be solved over the entire $z$ axis. Inside the cavity, the problem is much more complex since we have two coupled partial differential equations. In the special case of a mesa mode function $[u(z)=1$ inside the cavity, 0 elsewhere], the problem is, however, greatly simplified. In this case, inside the cavity, the operator $H-p^{2} / 2 m$ [see Eq. (1)] has normalized eigenstates $\left|\gamma_{n_{1}, n_{2}}^{\varepsilon}\right\rangle(\varepsilon=0, \pm)$ given by [16]

$$
\begin{gathered}
\left|\gamma_{n_{1}, n_{2}}^{0}\right\rangle=c_{b_{1}}^{0}\left|b_{1}, n_{1}+1, n_{2}\right\rangle+c_{b_{2}}^{0}\left|b_{2}, n_{1}, n_{2}+1\right\rangle, \\
\left|\gamma_{n_{1}, n_{2}}^{ \pm}\right\rangle=c_{a}^{ \pm}\left|a, n_{1}, n_{2}\right\rangle+c_{b_{1}}^{ \pm}\left|b_{1}, n_{1}+1, n_{2}\right\rangle+c_{b_{2}}^{ \pm}\left|b_{2}, n_{1}, n_{2}+1\right\rangle,
\end{gathered}
$$

with

$$
c_{b_{1}}^{0}=-\frac{\Omega_{n_{2}}}{\sqrt{\Omega_{n_{1}}^{2}+\Omega_{n_{2}}^{2}}}, \quad c_{b_{2}}^{0}=\frac{\Omega_{n_{1}}}{\sqrt{\Omega_{n_{1}}^{2}+\Omega_{n_{2}}^{2}}},
$$

and

$$
c_{a}^{ \pm}=\frac{-\Delta \pm \sqrt{\Delta^{2}+\Omega_{n_{1}}^{2}+\Omega_{n_{2}}^{2}}}{\beta^{ \pm}}, \quad c_{b_{\alpha}}^{ \pm}=\frac{\Omega_{n_{\alpha}}}{\beta^{ \pm}},
$$

where

$$
\beta^{ \pm}=\sqrt{2} \sqrt{\Omega_{n_{1}}^{2}+\Omega_{n_{2}}^{2}+\Delta\left(\Delta \mp \sqrt{\Delta^{2}+\Omega_{n_{1}}^{2}+\Omega_{n_{2}}^{2}}\right)} .
$$

The corresponding eigenvalues $\gamma_{n_{1}, n_{2}}^{\varepsilon}$ read

$$
\begin{gathered}
\gamma_{n_{1}, n_{2}}^{0}=E_{b}, \\
\gamma_{n_{1}, n_{2}}^{ \pm}=\frac{-\hbar \Delta \pm \hbar \sqrt{\Delta^{2}+\Omega_{n_{1}}^{2}+\Omega_{n_{2}}^{2}}+E_{b} .}{2} .
\end{gathered}
$$

In the basis $\left|\gamma_{n_{1}, n_{2}}^{\varepsilon}\right\rangle$ and in the $z$ representation, the Schrödinger equation takes the following form inside the cavity:

$$
i \hbar \frac{\partial}{\partial t} \psi_{n_{1}, n_{2}}^{\varepsilon}(z, t)=\left(-\frac{\hbar^{2}}{2 m} \frac{\partial^{2}}{\partial z^{2}}+V_{n_{1}, n_{2}}^{\varepsilon}\right) \psi_{n_{1}, n_{2}}^{\varepsilon}(z, t),
$$

with

$$
\psi_{n_{1}, n_{2}}^{\varepsilon}(z, t)=e^{i E_{b} t / \hbar}\left\langle z, \gamma_{n_{1}, n_{2}}^{\varepsilon} \mid \psi(t)\right\rangle
$$

and

$$
V_{n_{1}, n_{2}}^{\varepsilon}=\gamma_{n_{1}, n_{2}}^{\varepsilon}-E_{b}
$$

The most general solution of Eqs. (24) corresponding to the eigenstate $\left|\phi_{k}\right\rangle$ is given by

$$
\psi_{n_{1}, n_{2}}^{\varepsilon}(z, t)=e^{-i\left(\hbar k^{2} / 2 m\right) t} \varphi_{n_{1}, n_{2}}^{\varepsilon}(z),
$$

with

$$
\varphi_{n_{1}, n_{2}}^{\varepsilon}(z)=A_{n_{1}, n_{2}}^{\varepsilon} e^{i k_{n_{1}, n_{2}}^{\varepsilon} z}+B_{n_{1}, n_{2}}^{\varepsilon} e^{-i k_{n_{1}, n_{2}}^{\varepsilon} z},
$$

where $A_{n_{1}, n_{2}}^{\varepsilon}$ and $B_{n_{1}, n_{2}}^{\varepsilon}$ are complex coefficients and

$$
\left(k_{n_{1}, n_{2}}^{\varepsilon}\right)^{2}=k^{2}-\frac{2 m}{\hbar^{2}} V_{n_{1}, n_{2}}^{\varepsilon}
$$

We can write the wave function components of the atomfield state inside the cavity over the noncoupled state basis. The coefficients $\rho_{n_{1}, n_{2}}^{a}, \quad \rho_{n_{1}+1, n_{2}}^{b_{1}}, \quad \rho_{n_{1}, n_{2}+1}^{b_{2}}, \quad \tau_{n_{1}, n_{2}}^{a}, \quad \tau_{n_{1}+1, n_{2}}^{b_{1}}$, $\tau_{n_{1}, n_{2}+1}^{b_{2}}, A_{n_{1}, n_{2}}^{\varepsilon}$, and $B_{n_{1}, n_{2}}^{\varepsilon}$ are then found via the continuity conditions of the wave function and its first derivative at the cavity interfaces. For arbitrary values of the detuning $\Delta$ and in the mesa mode case, the obtained analytical expressions are too huge to be displayed here.

\section{Large-detuning limit}

For large detunings, the various probabilities (15)-(18) can be obtained in a much simpler way by use of the effective two-level Hamiltonian (3). In basis $\left|\gamma_{n_{1}, n_{2}}^{(i)}\right\rangle(i=1,2)$ given by

$$
\begin{aligned}
& \left|\gamma_{n_{1}, n_{2}}^{(1)}\right\rangle=c_{b_{1}}^{0}\left|b_{1}, n_{1}+1, n_{2}\right\rangle+c_{b_{2}}^{0}\left|b_{2}, n_{1}, n_{2}+1\right\rangle, \\
& \left|\gamma_{n_{1}, n_{2}}^{(2)}\right\rangle=c_{b_{2}}^{0}\left|b_{1}, n_{1}+1, n_{2}\right\rangle-c_{b_{1}}^{0}\left|b_{2}, n_{1}, n_{2}+1\right\rangle,
\end{aligned}
$$

and in the $z$ representation, the Schrödinger equation for the effective two-level Hamiltonian (3) takes the following form on the whole $z$ axis:

$$
i \hbar \frac{\partial}{\partial t} \psi_{n_{1}, n_{2}}^{(i)}(z, t)=\left(-\frac{\hbar^{2}}{2 m} \frac{\partial^{2}}{\partial z^{2}}+V_{n_{1}, n_{2}}^{(i)} u^{2}(z)\right) \psi_{n_{1}, n_{2}}^{(i)}(z, t),
$$

with

$$
\psi_{n_{1}, n_{2}}^{(i)}(z, t)=\left\langle z, \gamma_{n_{1}, n_{2}}^{(i)} \mid \psi(t)\right\rangle
$$

and

$$
\begin{gathered}
V_{n_{1}, n_{2}}^{(1)}=0, \\
V_{n_{1}, n_{2}}^{(2)}=\hbar \frac{\Omega_{n_{1}}^{2}+\Omega_{n_{2}}^{2}}{4 \Delta} .
\end{gathered}
$$

Equation (31) describes an elementary one-dimensional scattering problem for each component $\psi_{n_{1}, n_{2}}^{(i)}(z, t)$. The $\psi_{n_{1}, n_{2}}^{(1)}(z, t)$ components evolve like a free particle wave function whereas the $\psi_{n_{1}, n_{2}}^{(2)}(z, t)$ ones are similar to the wave function of a particle encountering the potential $V_{n_{1}, n_{2}}^{(2)} u^{2}(z)$ which is either a potential barrier or a potential well according to the sign of $\Delta$ (positive or negative, respectively) (see Fig. 2). This leads to the conclusion that, in the largedetuning limit, the three-level atom mazer does not mimic perfectly a two-level atom mazer for which the atom-cavity interaction reduces to the scattering of the atom upon a potential barrier and a potential well [2]. In this respect, the three-level atom mazer introduces new interesting options for engineering the interaction between the atoms and the cavity. It is also interesting to note that the scattering potential amplitude can be very easily tuned through the value of the detuning $\Delta$. With a two-level atom mazer, the potential 


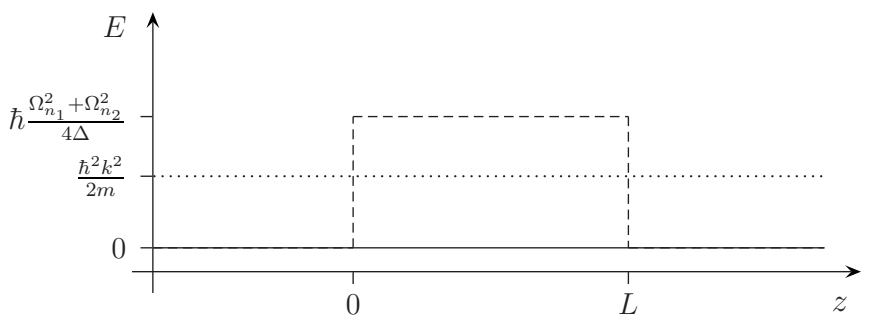

FIG. 2. Schematic representation of the energy $\hbar^{2} k^{2} / 2 m$ of an atom moving upon the micromaser cavity of length $L$. For large detunings, the cavity acts as a repulsive (or an attractive) potential (dashed curve) while one atomic component is freely transmitted (plain curve). The picture illustrates the case where the atom-field coupling is constant inside the cavity and the detuning $\Delta$ is positive (potential barrier).

amplitudes are fixed by the atom-cavity coupling constants and the cavity photon numbers.

If we again assume initially a monokinetic atom (with momentum $\hbar k$ ) coming upon the cavity from the left-hand side in the state $\left|b_{1}\right\rangle$ and the cavity field in the number state $\left|n_{1}+1, n_{2}\right\rangle$, the atom-field system is described by the two wave function components (which correspond to the eigenstate $\left|\phi_{k}\right\rangle$ of energy $\left.E_{k}=\hbar^{2} k^{2} / 2 m\right)$

$$
\psi_{n_{1}, n_{2}}^{(i)}(z, t)=e^{-i\left(\hbar k^{2} / 2 m\right) t} \varphi_{n_{1}, n_{2}}^{(i)}(z) \quad(i=1,2)
$$

with

$$
\varphi_{n_{1}, n_{2}}^{(1)}(z)=e^{i k z}
$$

and

$$
\varphi_{n_{1}, n_{2}}^{(2)}(z)= \begin{cases}e^{i k z}+\rho e^{-i k z}, & z<0 \\ \tau e^{i k z}, & z>L,\end{cases}
$$

where $\rho$ and $\tau$ are, respectively, the reflection and transmission coefficients of a particle of momentum $\hbar k$ coming upon the potential $V_{n_{1}, n_{2}}^{(2)} u^{2}(z)$ [see Eq. (31)]. Except for very particular cases, these coefficients are obtained numerically by solving the time-independent version of the Schrödinger equation (31). For a mesa mode, they are elementarily given by

$$
\begin{gathered}
\rho=i \Xi \sin \left(k^{\prime} L\right) \tau, \\
\tau=\left[\cos \left(k^{\prime} L\right)-i \Sigma \sin \left(k^{\prime} L\right)\right]^{-1},
\end{gathered}
$$

with

$$
\begin{aligned}
& k^{\prime}=\sqrt{k^{2}-\frac{\eta}{\widetilde{\Delta}} \kappa^{2},} \\
& \Xi=\frac{1}{2}\left(\frac{k^{\prime}}{k}-\frac{k}{k^{\prime}}\right), \\
& \Sigma=\frac{1}{2}\left(\frac{k^{\prime}}{k}+\frac{k}{k^{\prime}}\right),
\end{aligned}
$$

and

$$
\eta=\frac{\left(n_{1}+1\right)+\left(g_{1} / g_{2}\right)^{-2}\left(n_{2}+1\right)}{1+\left(g_{1} / g_{2}\right)^{-2}} .
$$

The initial wave vector reads

$$
\langle z \mid \psi(0)\rangle=e^{i k z}\left|b_{1}, n_{1}+1, n_{2}\right\rangle=e^{i k z}\left(c_{b_{1}}^{0}\left|\gamma_{n_{1}, n_{2}}^{(1)}\right\rangle+c_{b_{2}}^{0}\left|\gamma_{n_{1}, n_{2}}^{(2)}\right\rangle\right)
$$

and after the atom has left the interaction region, the initial state has evolved into

$$
\begin{aligned}
\langle z \mid \psi(t)\rangle= & e^{-i\left(\hbar k^{2} / 2 m\right) t}\left\{c_{b_{1}}^{0} e^{i k z} \theta(z-L)\left|\gamma_{n_{1}, n_{2}}^{(1)}\right\rangle+c_{b_{2}}^{0}\left[\rho e^{-i k z} \theta(-z)\right.\right. \\
& \left.\left.+\tau e^{i k z} \theta(z-L)\right]\left|\gamma_{n_{1}, n_{2}}^{(2)}\right\rangle\right\},
\end{aligned}
$$

where $\theta(z)$ is the Heaviside step function indicating that the atom is coming from the left. The wave function can be rewritten in the form

$$
\begin{aligned}
\langle z \mid \psi(t)\rangle= & e^{-i\left(\hbar k^{2} / 2 m\right) t} \\
& \times\left\{\left[r_{n_{1}+1, n_{2}}^{b_{1}} e^{-i k z} \theta(-z)\right.\right. \\
& \left.+t_{n_{1}+1, n_{2}}^{b_{1}} e^{i k z} \theta(z-L)\right]\left|b_{1}, n_{1}+1, n_{2}\right\rangle \\
& +\left[r_{n_{1}, n_{2}+1}^{b_{2}} e^{-i k z} \theta(-z)\right. \\
& \left.\left.+t_{n_{1}, n_{2}+1}^{b_{2}} e^{i k z} \theta(z-L)\right]\left|b_{2}, n_{1}, n_{2}+1\right\rangle\right\},
\end{aligned}
$$

with

$$
r_{n_{1}+1, n_{2}}^{b_{1}}=\frac{\Omega_{n_{1}}^{2}}{\Omega_{n_{1}}^{2}+\Omega_{n_{2}}^{2}} \rho,
$$

$$
t_{n_{1}+1, n_{2}}^{b_{1}}=\frac{\Omega_{n_{2}}^{2}}{\Omega_{n_{1}}^{2}+\Omega_{n_{2}}^{2}}+\frac{\Omega_{n_{1}}^{2}}{\Omega_{n_{1}}^{2}+\Omega_{n_{2}}^{2}} \tau,
$$

and

$$
\begin{gathered}
r_{n_{1}, n_{2}+1}^{b_{2}}=\frac{\Omega_{n_{1}} \Omega_{n_{2}}}{\Omega_{n_{1}}^{2}+\Omega_{n_{2}}^{2}} \rho, \\
t_{n_{1}, n_{2}+1}^{b_{2}}=\frac{\Omega_{n_{1}} \Omega_{n_{2}}}{\Omega_{n_{1}}^{2}+\Omega_{n_{2}}^{2}}(\tau-1) .
\end{gathered}
$$

The probabilities $\mathcal{P}_{b_{1} \rightarrow b_{1}}$ and $\mathcal{P}_{b_{1} \rightarrow b_{2}}$ are then, respectively, given by

$$
\mathcal{P}_{b_{1} \rightarrow b_{1}}=\left|r_{n_{1}+1, n_{2}}^{b_{1}}\right|^{2}+\left|t_{n_{1}+1, n_{2}}^{b_{1}}\right|^{2}
$$

and

$$
\mathcal{P}_{b_{1} \rightarrow b_{2}}=\left|r_{n_{1}, n_{2}+1}^{b_{2}}\right|^{2}+\left|t_{n_{1}, n_{2}+1}^{b_{2}}\right|^{2}
$$

\section{PROPERTIES OF THE TRANSITION PROBABILITIES}

In the following, we distinguish the hot and the cold atom regime. In the hot (respectively, cold) atom regime, the inci- 


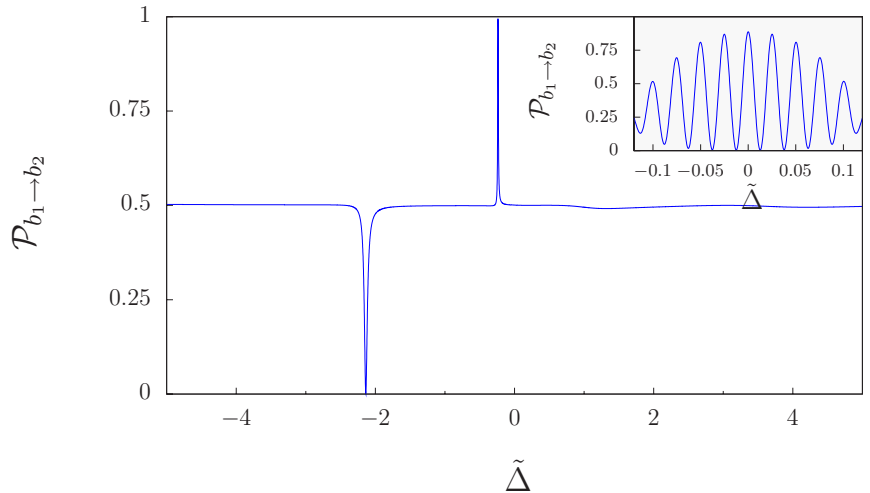

FIG. 3. (Color online) Transition probability $\mathcal{P}_{b_{1} \rightarrow b_{2}}$ with respect to the detuning $\widetilde{\Delta}$ for $k / \kappa=0.01, \kappa L=10, n_{1}=n_{2}=0$, and $g_{1} / g_{2}=1$. (Inset, $k / \kappa=100$ and $\kappa L=10^{5}$.)

dent kinetic energy of the atom is much larger (respectively, smaller) than the internal energies $V_{n_{1}, n_{2}}^{ \pm}$(for small $\Delta$ ) or $V_{n_{1}, n_{2}}^{(2)}($ for large $\Delta)$.

\section{A. Hot atom regime: Rabi limit}

In the hot atom regime, the atomic kinetic energy is such that the atoms are always transmitted through the cavity and the transition probabilities simplify to their expression obtained when the atomic motion is treated classically. We show this here below explicitly for a mesa mode and in the two extreme cases: at resonance $(\Delta=0)$ and in the fardetuning regime $\left(|\Delta| \gg \Omega_{n_{1}}, \Omega_{n_{2}}\right)$.

For a mesa mode, the atomic momenta inside the cavity associated to the corresponding $\varphi_{n_{1}, n_{2}}^{\varepsilon}(z)(\varepsilon=0, \pm)$ wave functions are given by

$$
\begin{gathered}
\hbar k_{n_{1}, n_{2}}^{0}=\hbar k \\
\hbar k_{n_{1}, n_{2}}^{ \pm} \simeq \hbar k\left(1+\frac{\kappa^{2}}{4 k^{2}}\left(\tilde{\Delta} \mp \sqrt{\left.\widetilde{\Delta}^{2}+4 \eta\right)}\right),\right.
\end{gathered}
$$

respectively.

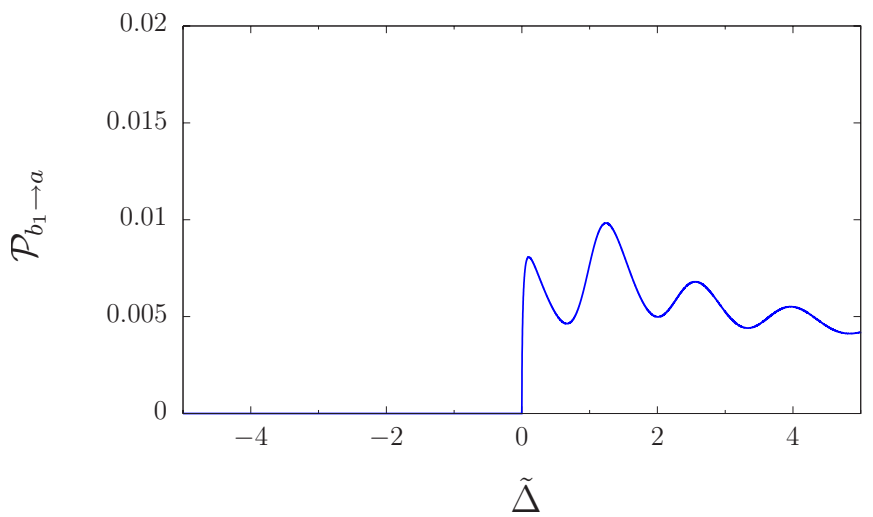

FIG. 4. (Color online) Transition probability $\mathcal{P}_{b_{1} \rightarrow a}$ with respect to the detuning $\widetilde{\Delta}$ for $k / \kappa=0.01, \kappa L=10, n_{1}=n_{2}=0$, and $g_{1} / g_{2}=1$.

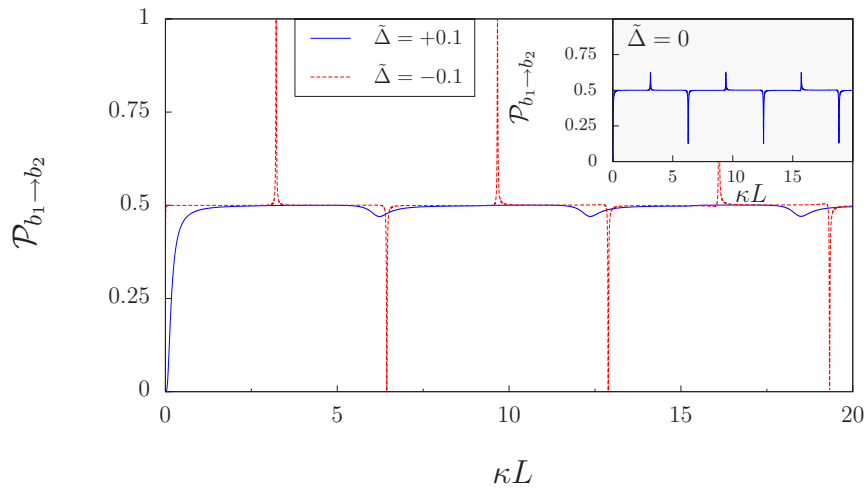

FIG. 5. (Color online) Transition probability $\mathcal{P}_{b_{1} \rightarrow b_{2}}$ with respect to the interaction length $\kappa L$ for $k / \kappa=0.01, \widetilde{\Delta}= \pm 0.1, n_{1}=n_{2}$ $=0$, and $g_{1} / g_{2}=1$. (Inset, $\widetilde{\Delta}=0$.)

At resonance, after the atom initially in the $\left|b_{1}\right\rangle$ state has interacted with the cavity field, the global state of the system reduces to

$$
\begin{aligned}
|\psi(t)\rangle= & \int d z \psi(z, t)\left(c_{b_{1}}^{0}\left|z, \gamma_{n_{1}, n_{2}}^{0}\right\rangle+c_{b_{1}}^{+} e^{-i\left(\kappa^{2} L / 2 k\right) \sqrt{\eta}}\left|z, \gamma_{n_{1}, n_{2}}^{+}\right\rangle\right. \\
& \left.+c_{b_{1}}^{-} e^{i\left(\kappa^{2} L / 2 k\right) \sqrt{\eta}}\left|z, \gamma_{n_{1}, n_{2}}^{-}\right\rangle\right)
\end{aligned}
$$

with

$$
\psi(z, t)=e^{i k z} e^{-i\left(\hbar k^{2} / 2 m\right) t} .
$$

The transition probability from state $\left|b_{1}\right\rangle$ to state $\left|b_{2}\right\rangle$ is thus given by

$$
\begin{aligned}
\mathcal{P}_{b_{1} \rightarrow b_{2}} & =\left|\left\langle z, b_{2}, n_{1}, n_{2}+1 \mid \psi(t)\right\rangle\right|^{2} \\
& =4 \frac{\Omega_{n_{1}}^{2} \Omega_{n_{2}}^{2}}{\left(\Omega_{n_{1}}^{2}+\Omega_{n_{2}}^{2}\right)^{2}} \sin ^{4}\left(\frac{\kappa^{2} L}{4 k} \sqrt{\eta}\right)
\end{aligned}
$$

which can be rewritten

$$
\mathcal{P}_{b_{1} \rightarrow b_{2}}=4 \frac{\Omega_{n_{1}}^{2} \Omega_{n_{2}}^{2}}{\left(\Omega_{n_{1}}^{2}+\Omega_{n_{2}}^{2}\right)^{2}} \sin ^{4}\left(\frac{\Omega \tau}{2}\right)
$$

with

$$
\Omega=\frac{1}{2} \sqrt{\Omega_{n_{1}}^{2}+\Omega_{n_{2}}^{2}},
$$

where $\tau=\frac{m L}{\hbar k}$ is the classical transit time of the thermal atoms through the cavity. Equation (56) is exactly the classical expression of the transition probability from state $\left|b_{1}\right\rangle$ to state $\left|b_{2}\right\rangle$ for a three-level atom interacting with a bimodal cavity during a time $\tau[16]$.

We get a similar result for the transition probability from state $\left|b_{1}\right\rangle$ to state $|a\rangle$, 


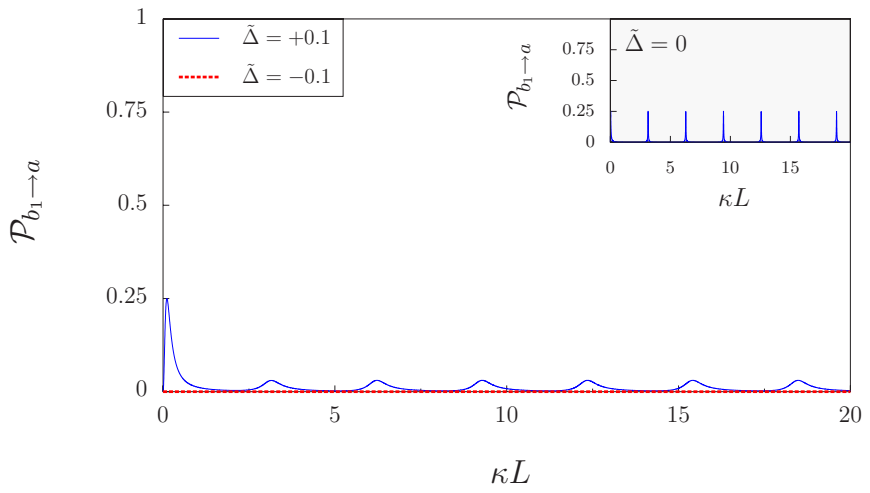

FIG. 6. (Color online) Transition probability $\mathcal{P}_{b_{1} \rightarrow a}$ with respect to the interaction length $\kappa L$ for $k / \kappa=0.01, n_{1}=n_{2}=0, g_{1} / g_{2}=1$, and $\widetilde{\Delta}= \pm 0.1$. (Inset, $\widetilde{\Delta}=0$.)

$$
\mathcal{P}_{b_{1} \rightarrow a}=\left|\left\langle z, a, n_{1}, n_{2} \mid \psi(t)\right\rangle\right|^{2}=\frac{\Omega_{n_{1}}^{2}}{\Omega_{n_{1}}^{2}+\Omega_{n_{2}}^{2}} \sin ^{2}\left(\frac{\kappa^{2} L}{2 k} \sqrt{\eta}\right),
$$

which again reduces to the classical expression of this probability [16],

$$
\mathcal{P}_{b_{1} \rightarrow a}=\frac{\Omega_{n_{1}}^{2}}{\Omega_{n_{1}}^{2}+\Omega_{n_{2}}^{2}} \sin ^{2}(\Omega \tau) .
$$

For large detunings $\left(|\Delta| \gg \Omega_{n_{1}}, \Omega_{n_{2}}\right)$, the global state of the system can be written after the atom-field interaction

$$
|\psi(t)\rangle \simeq \int d z \psi(z, t)\left(c_{b_{1}}^{0}\left|z, \gamma_{n_{1}, n_{2}}^{0}\right\rangle+c_{b_{1}}^{+} e^{-i\left(\kappa^{2} L / 2 k\right) \eta / \tilde{\Delta}}\left|z, \gamma_{n_{1}, n_{2}}^{+}\right\rangle\right)
$$

since $c_{b_{1}}^{-} \simeq 0$ [see Eqs. (21) and (22)]. The transition probability from state $\left|b_{1}\right\rangle$ to state $\left|b_{2}\right\rangle$ is thus given by

$$
\begin{aligned}
\mathcal{P}_{b_{1} \rightarrow b_{2}} & =\left|\left\langle z, b_{2}, n_{1}, n_{2}+1 \mid \psi(t)\right\rangle\right|^{2} \\
& =4 \frac{\Omega_{n_{1}}^{2} \Omega_{n_{2}}^{2}}{\left(\Omega_{n_{1}}^{2}+\Omega_{n_{2}}^{2}\right)^{2}} \sin ^{2}\left(\frac{\kappa^{2} L}{4 k} \eta / \tilde{\Delta}\right) .
\end{aligned}
$$

It is then enough to notice that Eq. (61) can be cast in the form

$$
\mathcal{P}_{b_{1} \rightarrow b_{2}}=\frac{\Omega_{\text {eff }}^{2}}{\Omega_{\text {eff }}^{2}+\Delta_{\text {eff }}^{2}} \sin ^{2}\left(\sqrt{\Omega_{\text {eff }}^{2}+\Delta_{\text {eff }}^{2}} \frac{\tau}{2}\right)
$$

with

$$
\Delta_{\text {eff }}=\frac{\Omega_{n_{2}}^{2}-\Omega_{n_{1}}^{2}}{4 \Delta}, \quad \Omega_{\text {eff }}=\frac{\Omega_{n_{1}} \Omega_{n_{2}}}{2 \Delta} .
$$

Equation (62) is exactly the transition probability of a two-level system $\left|b_{1}\right\rangle$ and $\left|b_{2}\right\rangle$ interacting during a time $\tau$ with a monomodal cavity and with a detuning $\Delta_{\text {eff }}$ and a Rabi frequency $\Omega_{\text {eff }}$. It is well known that this is precisely the

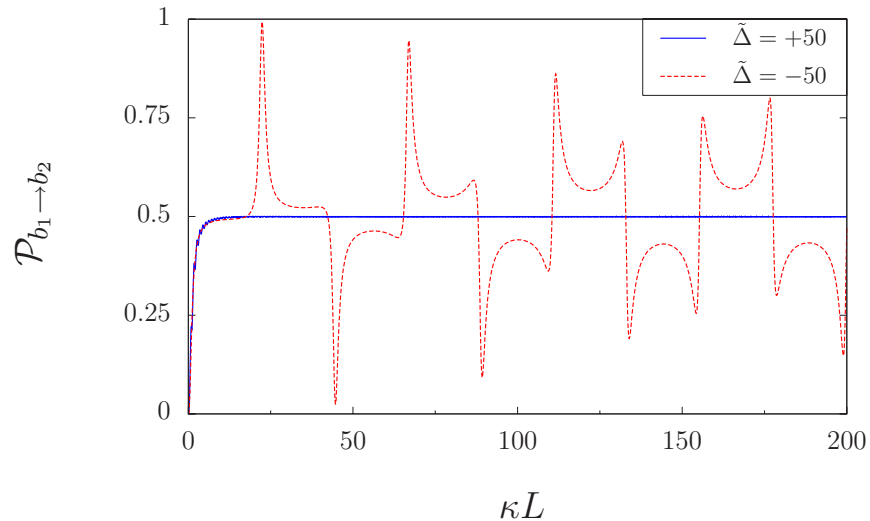

FIG. 7. (Color online) Transition probability $\mathcal{P}_{b_{1} \rightarrow b_{2}}$ with respect to the interaction length $\kappa L$ for $k / \kappa=0.01, \widetilde{\Delta}= \pm 50, n_{1}=n_{2}$ $=0$, and $g_{1} / g_{2}=1$.

behavior of a three-level system interacting during a time $\tau$ with a bimodal cavity in the far-detuning regime and in the two-photon resonance condition $[17,19,20]$.

\section{B. Cold atom regime}

In the cold atom regime, the properties of the atomic transition probabilities differ significantly from the hot atom regime where the Rabi oscillatory behavior is observed with respect to the cavity length $L$ [see Eqs. (55) and (61)]. The specific properties of a bimodal cavity pumped by cold threelevel atoms were already pointed out at resonance by Arun et al. [12]. Out of resonance, other specific properties are exhibited by the system.

First, and in contrast to the hot atom regime, the transition probabilities are sensitive to the sign of the detuning. This is illustrated in Figs. 3 and 4 in the case of a mesa mode function for the probabilities $\mathcal{P}_{b_{1} \rightarrow b_{2}}$ and $\mathcal{P}_{b_{1} \rightarrow a}$, respectively. For negative detunings, as discussed in Sec. II, the transition probability $\mathcal{P}_{b_{1} \rightarrow a}$ vanishes very quickly (see Fig. 4).

Second, the transition probability $\mathcal{P}_{b_{1} \rightarrow b_{2}}$ exhibits in the cold atom regime very fine resonances with respect to the interaction length $\kappa L$ that are amplified by use of a small negative detuning (see Fig. 5). Rather, for small positive detunings, these resonances are significantly decreased. About the transition probability $\mathcal{P}_{b_{1} \rightarrow a}$, the fine resonances that are observed in absence of detuning decrease significantly by use of a small positive detuning, whereas they are annihilated for small negative detunings (see Fig. 6). In this case, the three-level atom mazer behaves like a two-level atom one even if the system is not running in the far-detuning regime. We then get in the strong coupling regime a virtual two-level atom mazer without spontaneous emission between the two levels $\left|b_{1}\right\rangle$ and $\left|b_{2}\right\rangle$. We eliminate this way a major issue of the conventional two-level atom mazer and parameters like those of Fig. 5 could be advantageously used to test the specific properties of the micromaser in the cold atom regime. With these parameters, the resonances are well peaked and reach the value 1 while those of the usual two-level atom mazer are limited to 0.5 [1]. 


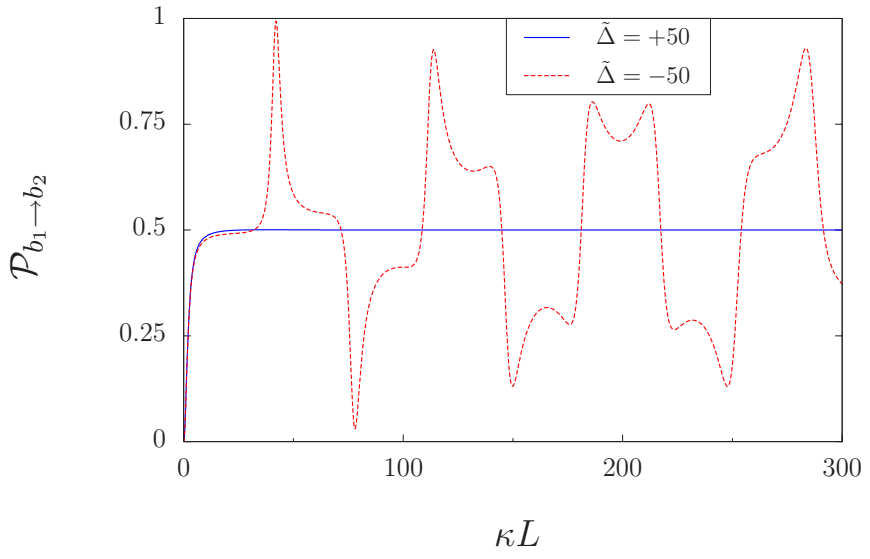

FIG. 8. (Color online) Transition probability $\mathcal{P}_{b_{1} \rightarrow b_{2}}$ with respect to the interaction length $\kappa L$ for a sine mode $u(z)=\sin (\pi z / L)$ for $k / \kappa=0.01, \widetilde{\Delta}= \pm 50, n_{1}=n_{2}=0$, and $g_{1} / g_{2}=1$.

In the large detuning regime, the resonances are much less peaked, especially as the cavity mode gets smoother (see Figs. 7 and 8 for a mesa and a sinusoidal mode). When positive detunings are used, the transition probability $\mathcal{P}_{b_{1} \rightarrow b_{2}}$ very rapidly reaches a plateau at a value $\mathcal{P}$ given by

$$
\mathcal{P}=2 \frac{\Omega_{n_{1}}^{2} \Omega_{n_{2}}^{2}}{\left(\Omega_{n_{1}}^{2}+\Omega_{n_{2}}^{2}\right)^{2}} .
$$

This is true for all modes. Indeed, for cold atoms and positive detunings, $|\rho| \simeq 1$ and $\tau \simeq 0$. Inserting those values into Eqs. (48), (49), and (51) yields Eq. (64).

\section{EXPERIMENTAL CONSIDERATIONS}

We now discuss how our proposal could be experimentally implemented with present technology. The apparatus of Hood et al. [21,22] yield a microlaser cavity with a Gaussian mode waist of $15 \mu \mathrm{m}$ and a free spectral range (FSR) of the order of $7 \mathrm{THz}$. Sending cold metastable xenon atoms with no nuclear spin [27] through a similar cavity would define a $\Lambda$-configuration system with a coupling strength $g / 2 \pi$ of the order of $75 \mathrm{MHz}$ via the two transitions $6 s[3 / 2]_{2}^{o}-6 p[5 / 2]_{2}$ and $6 s[3 / 2]_{1}^{o}-6 p[5 / 2]_{2}$ at 904.54 and $992.32 \mathrm{~nm}$, respectively [23]. These two transitions are separated by a frequency difference of $29.2 \mathrm{THz}$ that can match 4 FSR by tuning properly the cavity length. The far-detuning regime would be easily obtained while remaining far below the free spectral range (we have $\Delta / g=100$ for $\Delta / 2 \pi \simeq 7.5 \mathrm{GHz}$ $\ll$ FSR). For a detuning $\Delta / g$ of 1 (respectively, 10 and 100), the cold atom regime is reached at a translational motion temperature of $3 \mathrm{mK}$ (respectively, $0.5 \mathrm{mK}$ and $51 \mu \mathrm{K}$ ). With atoms launched horizontally like in our scheme of Fig. 1 (using, e.g., the conveyor belt technique [24]), such temperatures would produce a vertical fall during the atomic transit time through the cavity mode waist of the order of $2.8 \mathrm{~nm}$ (respectively, $17 \mathrm{~nm}$ and $0.17 \mu \mathrm{m}$ ). These falls are so small in comparison with the size of the mode waist that the gravity action during the atom-cavity interaction could be neglected. All these parameters show that an experiment exhibiting the effects described in this paper is accessible with present technology in the optical domain. Also, this domain has the advantage of being thermal photon free. We would not recommend working in the microwave domain since the coupling strengths (and thus the maximal allowed translational temperatures defining the cold atom regime) are usually much smaller there. Also the cavity mode sizes are much larger and the gravity action could not be considered anymore as negligible. A model including gravity and thermal photons as shown in Ref. [25] for two-level atoms would be necessary in this case.

\section{SUMMARY}

In this paper, we have analyzed the quantum theory of the two-mode micromaser pumped by cold three-level atoms with a $\Lambda$ configuration under the two-photon resonance condition. We have focused more specifically on the largedetuning limit and have shown that this regime can mimic a virtual two-level atom micromaser without spontaneous emission where the cavity acts for the atoms as a potential barrier or a potential well (depending on the sign of the detuning) and a zero potential but not as a potential barrier and a potential well as it is the case for the usual cold twolevel atom mazer. This introduces interesting options for engineering the interaction between the cold atoms and the cavity. The elimination of the spontaneous emission solves a major issue of the conventional two-level atom mazer as the transit times of cold atoms inside and in the vicinity of the cavity is usually much larger than the atomic level lifetimes.

We have also shown that the cold atom regime is very sensitive to the sign of the detuning (in contrast to the hot atom regime) and that, according to this sign, the cavity may speed up or slow down the incident three-level atoms after their interaction with the field.

\section{ACKNOWLEDGMENT}

One of the authors (J.M.) thanks the Belgian FRIA for financial support.
[1] M. O. Scully, G. M. Meyer, and H. Walther, Phys. Rev. Lett. 76, 4144 (1996).

[2] B.-G. Englert, J. Schwinger, A. O. Barut, and M. O. Scully, Europhys. Lett. 14, 25 (1991).

[3] T. Bastin and J. Martin, Phys. Rev. A 67, 053804 (2003).
[4] G. M. Meyer, M. O. Scully, and H. Walther, Phys. Rev. A 56, 4142 (1997).

[5] M. Löffler, G. M. Meyer, M. Schröder, M. O. Scully, and H. Walther, Phys. Rev. A 56, 4153 (1997).

[6] M. Schröder, K. Vogel, W. P. Schleich, M. O. Scully, and H. 
Walther, Phys. Rev. A 56, 4164 (1997).

[7] Z.-M. Zhang and L.-S. He, Opt. Commun. 157, 77 (1998).

[8] Z.-M. Zhang, S.-W. Xie, Y.-L. Chen, Y.-X. Xia, and S.-K. Zhou, Phys. Rev. A 60, 3321 (1999).

[9] Z.-M. Zhang, S.-W. Xie, and S.-K. Zhou, J. Phys. B 32, 4013 (1999).

[10] Z.-M. Zhang, S.-W. Xie, W.-Q. Liang, and S.-K. Zhou, J. Phys. B 33, 2125 (2000).

[11] W.-Q. Liang, Z.-Q. Niu, Z.-M. Zhang, and S.-W. Xie, J. Phys. B 34, 4427 (2001).

[12] R. Arun, G. S. Agarwal, M. O. Scully, and H. Walther, Phys. Rev. A 62, 023809 (2000).

[13] R. Arun and G. S. Agarwal, Phys. Rev. A 66, 043812 (2002).

[14] A.-S. F. Obada and M. Abdel-Aty, Int. J. Mod. Phys. B 17, 2735 (2003).

[15] M. Abdel-Aty and A.-S. F. Obada, J. Phys. B 36, 3173 (2003).

[16] H. I. Yoo and J. H. Eberly, Phys. Rep. 118, 239 (1985).
[17] C. C. Gerry and J. H. Eberly, Phys. Rev. A 42, 6805 (1990).

[18] J. J. Hope and C. M. Savage, Phys. Rev. A 53, 1697 (1996).

[19] M. Alexanian and S. K. Bose, Phys. Rev. A 52, 2218 (1995).

[20] N. V. Vitanov and S. Stenholm, Phys. Rev. A 55, 648 (1997).

[21] C. J. Hood, M. S. Chapman, T. W. Lynn, and H. J. Kimble, Phys. Rev. Lett. 80, 4157 (1998).

[22] C. J. Hood, T. W. Lynn, A. C. Doherty, A. S. Parkins, and H. J. Kimble, Science 287, 1447 (2000).

[23] J. Sabbagh and N. Sadeghi, J. Quant. Spectrosc. Radiat. Transf. 17, 297 (1977).

[24] S. Nußmann, M. Hijlkema, B. Weber, F. Rohde, G. Rempe, and A. Kuhn, Phys. Rev. Lett. 95, 173602 (2005).

[25] T. Bastin and J. Martin, Phys. Rev. A 72, 053815 (2005).

[26] M. Walhout, H. J. L. Megens, A. Witte, and S. L. Rolston, Phys. Rev. A 48, R879 (1993).

[27] Metastable xenon can be cooled down via the usual laser cooling techniques, see, e.g., Ref. [26]. 